\title{
SAND99-1545C
}

\section{Strong Resonant Intersubband Magnetopolaron Effect in Heavily Modulation- Doped GaAs/AlGaAs Single Quantum Wells at High Magnetic Fields}

Y.J. Wang ${ }^{1}$, Y.A. Leem ${ }^{1}$, B. D. McCombe ${ }^{2}$, X.-G. Wu ${ }^{3}$, F.M. Peeters ${ }^{4}$, E. Jones ${ }^{5}$, J. Reno ${ }^{5}$, X.Y. Lee ${ }^{6}$, and H.W. Jiang ${ }^{6}$

${ }^{1}$ National High Magnetic Field Lab at Florida State University, Tallahassee, FL 32310, USA

${ }^{2}$ Department of Physics, State University of New York at Buffalo, Buffalo, NY 14260, USA

${ }^{3}$ National Lab for Superlattices and Microstructures Institute of Semiconductors, Beijing, China

${ }^{4}$ Department Natuurkunde, University of Antwerpen, (UIA), B-2610 Antwerpen, Belgium

${ }^{5}$ Sandia National Laboratories, Albuquerque, NM 87185-0601, USA

${ }^{6}$ Department of Physics and Astronomy, University of California at Los Angels, CA 90095, USA

Electron cyclotron resonance (CR) has been studied in magnetic fields up to $32 \mathrm{~T}$ in two heavily modulation-d-doped $\mathrm{GaAs} / \mathrm{Al}_{0.3} \mathrm{Ga}_{0.7} \mathrm{As}$ single-quantum-well. Little effect on electron CR is observed in either sample in the region of resonance with the GaAs LO phonons. However, above $\mathrm{w}_{\mathrm{LO}}$ at $\mathrm{B}>27 \mathrm{~T}$, electron $\mathrm{CR}$ exhibits a strong avoided-levelcrossing splitting for both samples at energies close to $E_{L O}+\left(E_{2}-E_{1}\right)$, where $E_{2}$, and $E_{1}$ are the energies of the bottoms of the second and the first subbands, respectively. The energy separation between the two branches is large, reaching a minimum of about $40 \mathrm{~cm}^{-}$ ${ }^{1}$ around $30.5 \mathrm{~T}$ for both samples. This splitting is due to a three-level resonance between the second LL of the first electron subband and the lowest LL of the second subband plus a LO phonon. The large splitting in the presence of high electron densities is due to the absence of occupation (Pauli-principle) effects in the final states and weak screening for this three level process.

PACS codes: 71.38.+I, 71.70.Di, 78.20.Ls

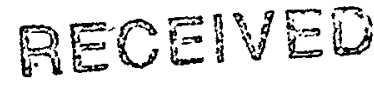

AUG 249989

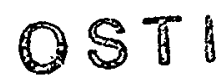

Key words: Electron Cyclotron Resonance, Electron-Phonon Interaction, Subband

Corresponding author:

Y.J. Wang

National High Magnetic Field Laboratory at Florida State University

1800 East Paul Dirac Drive

Tallahassee, Florida 32310

(tel): $1-850-644-1496$

(fax): 1-850-644-0534

e-mail: wang@magnet.fsu.edu

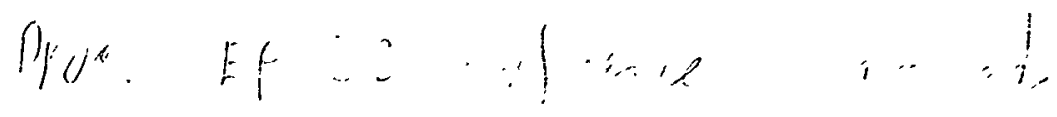




\section{DISCLAIMER}

This report was prepared as an account of work sponsored by an agency of the United States Government. Neither the United States Government nor any agency thereof, nor any of their employees, make any warranty, express or implied, or assumes any legal liability or responsibility for the accuracy, completeness, or usefulness of any information, apparatus, product, or process disclosed, or represents that its use would not infringe privately owned rights. Reference herein to any specific commercial product, process, or service by trade name, trademark, manufacturer, or otherwise does not necessarily constitute or imply its endorsement, recommendation, or favoring by the United States Government or any agency thereof. The views and opinions of authors expressed herein do not necessarily state or reflect those of the United States Government or any agency thereof. 


\section{DISCLAIMER}

Portions of this document may be illegible in electronic image products. Images are produced from the best available original document. 
The interaction of electrons with optical phonons in quasi-two dimensional (Q2D) systems has been studied both theoretically [1] and experimentally [2] for a number of years because of its importance with respect to both device application and fundamental physics of these systems. Recently, due to the availability of sufficient high magnetic fields, new, and interesting phenomena associated with electron-phonon (e-ph) interaction have been observed [3]. For polar GaAs/AlGaAs bulk materials, the dominant interaction is between the electrons and longitudinal optical (LO) phonons. For a Q2D system, this interaction (polaron effect) can affect both free electron cyclotron resonance (CR) and intra-band impurity transition. When a pair of unperturbed electron energy levels, e.g., $\mathrm{N}=0$ to $\mathrm{N}=1 \mathrm{LL}$, or 1 s to $2 \mathrm{p}+$ impurity levels, has energy separation close to $\mathrm{E}_{\mathrm{Lo}}$, the LOphonon energy, a two-level resonant magnetopolaron effect occurs. For intra-band impurity transitions, when magnetic field is so high that the energy separation of the $2 \mathrm{P}^{+}$and $2 \mathrm{P}^{-}$states is close to $\mathrm{E}_{\mathrm{Lo}}$, it has been shown [4] that a three-level magnetopolaron effect occurs. In this case an electron is excited from the ground (1S) state to the $2 \mathrm{P}^{+}$state, which is resonantly coupled to the $2 \mathrm{P}^{+}$state via emission of an LO phonon.

The two-level magnetopolaron effect has been observed in electron CR measurements, but three-level effects in electron $C R$ measurements have not been reported. Recently, our theoretical calculations have predicted that three-level coupling should affect the ECR excitation spectrum greatly at fields high enough to involve the two different subbands in the three-level process. Similar to the impurity system, a three-level resonance should occur when magnetic field is such that the energy difference between a higher LL of the first subband and a lower LL of the second subband is equal to $\mathrm{E}_{\mathrm{LO}}$ for GaAs. In the electron $C R$ process a free electron is excited from the $N=0 L L$ in the lowest subband $\left(E_{1}\right)$ to the $N=1 \mathrm{LL}$ in the same subband via the electric dipole interaction; the final state of this transition is coupled resonantly via the emission of an LO phonon to the $\mathrm{N}=0 \mathrm{LL}$ of the second subband $\left(\mathrm{E}_{2}\right)$.

To study this three-level resonant magnetopolaron effect and the effects of a high density of free electrons we have measured electron $C R$ in two heavily modulation-ddoped $\mathrm{GaAs} / \mathrm{Al}_{0.3} \mathrm{Ga}_{0.7} \mathrm{As} 240 \AA$ single-quantum-well samples in magnetic fields up to 32 $T$. In the two-level resonant magnetopolaron region $\left(\mathrm{E}_{\mathrm{CR}}=\mathrm{E}_{\mathrm{LO}}\right)$ there is little effect on ECR in either sample. The LL occupation (Pauli-principle) effects and the strong screening of the e-ph interaction for non-integer filling factors combine to reduce the effective interaction. However, above the GaAs LO phonon frequency at high fields (B > $27 \mathrm{~T}$ ), electron $\mathrm{CR}$ exhibits a very strong avoided-level-crossing splitting for both samples at energies close to $E_{L O}+\left(E_{2}-E_{1}\right)$. The splitting between the two branches is large, with a minimum energy separation of about $40 \mathrm{~cm}^{-1}$ near $30.5 \mathrm{~T}$ for both samples. This splitting is attributed to the three-level resonance discussed above.

Since these two samples are very heavily-doped, occupancy and electron screening effects should be very strong. This is the reason for the absence of an observable direct resonant interaction (two level system) between the electrons and GaAs LO phonons at lower fields and photon energies. For a three-level resonance, the Pauli-principle (occupation effect) plays no role, because the final state is essentially unoccupied - it lies in the next (unoccupied at high fields) subband. In addition, screening is also weak, because the single particle wave functions in the $\mathrm{z}$-direction are orthogonal for the first and second subbands. Therefore the screening of states in the second subband due to electrons in the lowest subband is expected to be weak. This basic physics accounts for the large splitting 
observed for the three level system and the absence of an observable effect for the two level system.

The far infrared transmission measurements were carried out in the National High Magnetic Field Laboratory at Florida State University. The set-up consists of a Brüker $113 \mathrm{v}$ Fourier transform spectrometer in conjunction with a metal light-pipe condensingcone system (details are given elsewhere [5]), and a $4.2 \mathrm{~K}$ silicon bolometer. The temperature of the sample is maintained at $4.2 \mathrm{~K}$, and the applied field is up to $32 \mathrm{~T}$. The two $\mathrm{GaAs} / \mathrm{Al}_{.3} \mathrm{Ga}_{.7} \mathrm{As}$ single quantum well samples were grown by molecular beam epitaxy at Sandia National Laboratories. The well width for both samples is $240 \AA$, and both samples are d-doped with silicon in the barrier with $240 \AA$ set-off distance. The electron densities measured from the quantum Hall effect and longitudinal resistivity are $8.0 \times 10^{11} \mathrm{~cm}^{-2}$, and $8.9 \times 10^{11} \mathrm{~cm}^{-2}$, respectively for samples 1 and 2 . The nominal doping concentrations for the two samples are $2 \times 10^{12} \mathrm{~cm}^{-2}$ and $4 \times 10^{12} \mathrm{~cm}^{-2}$, which are very different from the measured electron densities. There are two possible reasons for the discrepancy. 1) Quantum Hall effect measurements are dominated by the lowest subband occupancy. 2) Because of heavily-doping, band banding for our samples is very large, this can bring the donor level in the barrier down to the chemical potential, leading to some neutral donors in the barrier and causing parallel conduction.

Figure 1 is a plot of the measured electron CR frequency vs. magnetic field for the two samples. The solid lines are the calculated single particle electron CR transition frequencies for the $\mathrm{N}=0$ to $\mathrm{N}=1$ transition (ignoring spin) of the ground subband including non-parabolicity. These calculations do not consider the e-ph interaction. At low magnetic fields, there is no significant deviation from the single particle calculation. At higher fields a small splitting is observed, and there is some deviation of one of the two peaks to lower energies when the electron CR energy approaches that of the GaAs LO phonon (e.g. see Fig. 1a) above $10 \mathrm{~T}$ ). The splitting results from a combination of self consistent band bending, occupancy of LLs in the second subband in this field region, and non-parabolicity. [6] The deviation of the lower line at fields above $15 \mathrm{~T}$ may be due to the (two-level) resonant magnetopolaron effect for a small density of electrons occupying the lowest LL of the second subband in this region. Further detailed analysis is needed to substantiate this assertion. Apart from this effect in the lower density sample, in the region of resonance with the GaAs LO phonons $\left(E_{C R}=E_{L O}\right)$, little effect on $C R$ is observed in either sample. However, above the GaAs LO phonon frequency at high fields ( $\mathrm{B}>27 \mathrm{~T}$ ), electron CR exhibits a very strong avoided-level-crossing splitting for both samples. The pinning frequency is slightly different for the two samples, about $400 \mathrm{~cm}^{-1}$ for sample 1 , and $395 \mathrm{~cm}^{-1}$ for sample 2 . The subband separations obtained from our self-consistently calculations are $12.9 \mathrm{mev}$ and $12.2 \mathrm{mev}$ for samples 1 and 2, respectively. The summation of the calculated subband energy and the GaAs LO phonon energy gives rise to a resonant frequency which is almost identical to the pinning frequency observed in the experiment for both samples.

Figure 2 is the raw magnetotransmission spectra for these two samples at $30 \mathrm{~T}$ and $32 \mathrm{~T}$. These spectra are normalized magnetotransmission, ratios of a spectrum taken at magnetic field to a spectrum taken at zero field. It is clear that both samples show similar behavior. When the two branches are first observable, the lower energy branch is definitely the dominant one. Above the resonant field, (about $30.5 \mathrm{~T}$ ) or energy, the upper branch becomes dominant. The lower energy branch loses intensity gradually as the field is 
increased, while the higher energy branch gains intensity over the same region. This is typical behavior for the resonant magnetopolaron effect.

Several possible origins for this splitting have been considered. Since the resonance region is very close the LO phonon frequency of AlAs, the possibility of this splitting being from the interaction between electron and some kind of AlAs LO-phonon modes has been considered. There are two possible scenarios in which electrons can interact significantly with AlAs LO-phonon modes [3]: 1) electrons in the wells can interact with the AlAs-like slab LO phonons in the barriers via penetration of the electronic envelope function into the barriers, or 2) electrons in the wells can interact with AlAs-like interface phonons which have displacements that exist in the wells. Both scenarios are easily excluded based on arguments similar to those presented in ref. [3] Basically, for such wide wells $(240 \AA)$ the calculated splittings are very small. As stated previously, we believe that this splitting is due to a three-level resonance between the second LL of the first electron subband and the lowest $\mathrm{LL}$ of the second subband plus a $\mathrm{LO}$ phonon (at an energy of $\left.E_{L O}+\left(E_{2}-E_{1}\right)\right)$. Our self-consistent calculation of electron $C R$, which includes $\mathrm{e}-\mathrm{ph}$ interactions and the influence on the e-ph interaction from LL occupancy, electron screening, and band nonparabolicity, has predicted this three-level resonance. However, the large contrast between the substantial energy splitting for the three-level resonance, and the almost non-existent effect in the two-level resonance region was not anticipated. This interesting behavior has been understood based on the fact that electron screening and occupancy effects are very strong in the two level system, and very weak in the three level system. These qualitative arguments account for the large splitting observed for the three level system (with essentially NO splitting observed for the two level system).

To test the above arguments quantitatively, detailed calculations specifically for our two sample structures have been carried out to compare with the experimental results. Details of the general calculation is given in ref. [6]. In the self-consistent subband calculation, the center of the quantum well is taken as the zero of the Hartree potential, and the d-doping in the barrier is assumed to be symmetric about the quantum well, each doping spike contributing half of its the electrons to adjacent wells. The conduction band non-parabolicity is taken into account via the simple two-band Kane model. The e-ph interaction and the influence of LL occupancy and screening on it are included in the calculation. The comparison between the calculated results and the experimental results is shown in Fig. 3 . The agreement between the experimental results and theoretical calculations is very good over the entire field region for both samples. This provides quantitative confirmation of the qualitative arguments given above.

We have clearly demonstrated the importance of electron occupation and screening effects for the e-ph interaction. Certain e-ph interaction can be greatly reduced by high electron densities in the system. This effect can be possibly used for making some quantum well devices with high electron densities.

High field transmission measurement was performed at the National High Magnetic Field Laboratory, which is supported by NSF Cooperative Agreement No. DMR9016241 and by the State of Florida. Sandia is a multiprogram laboratory operated by Sandia Corporation, a Lockheed Martin Company, for the United States Department of Energy under contract DE-AC04-94AL85000. 


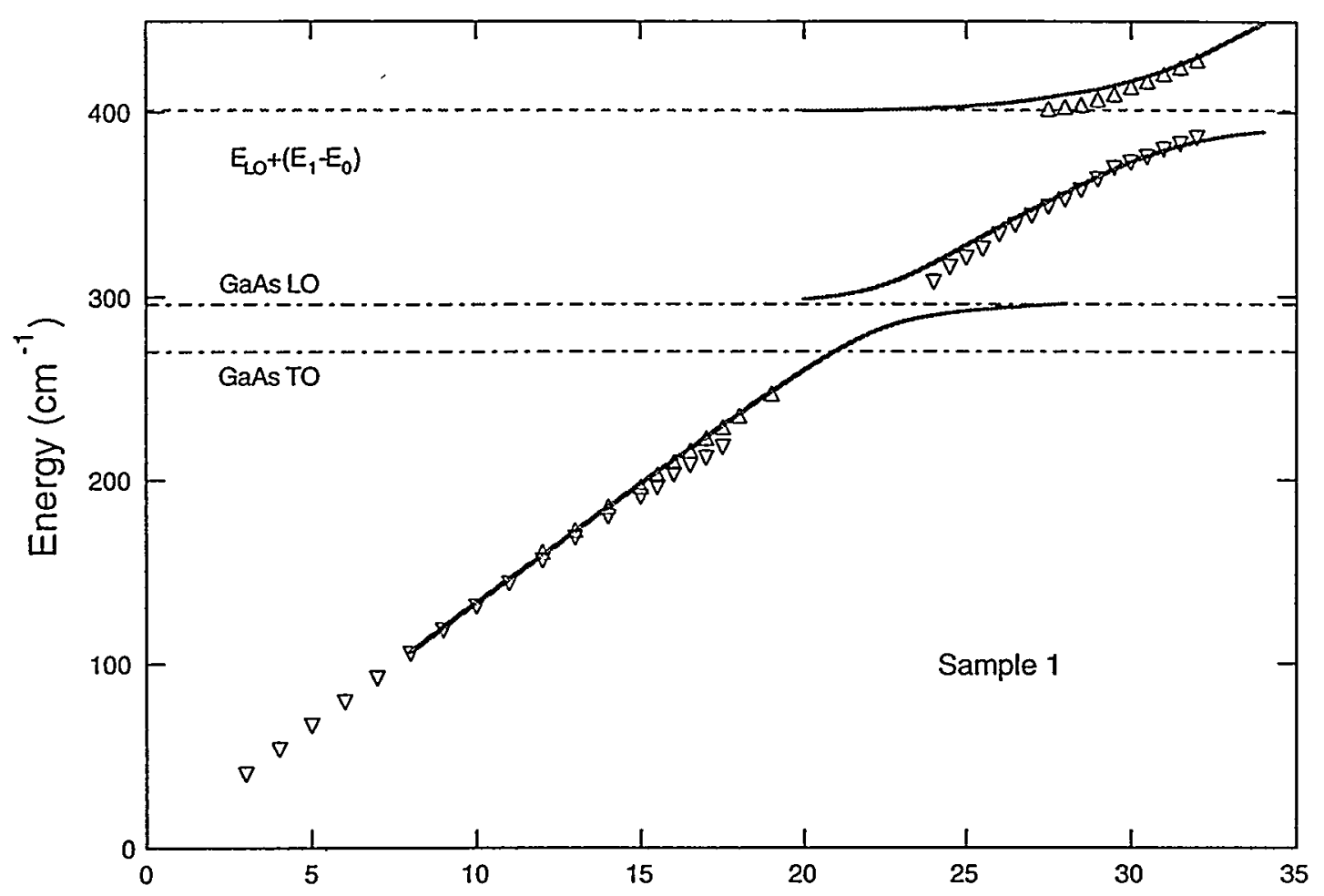

Field $(T)$

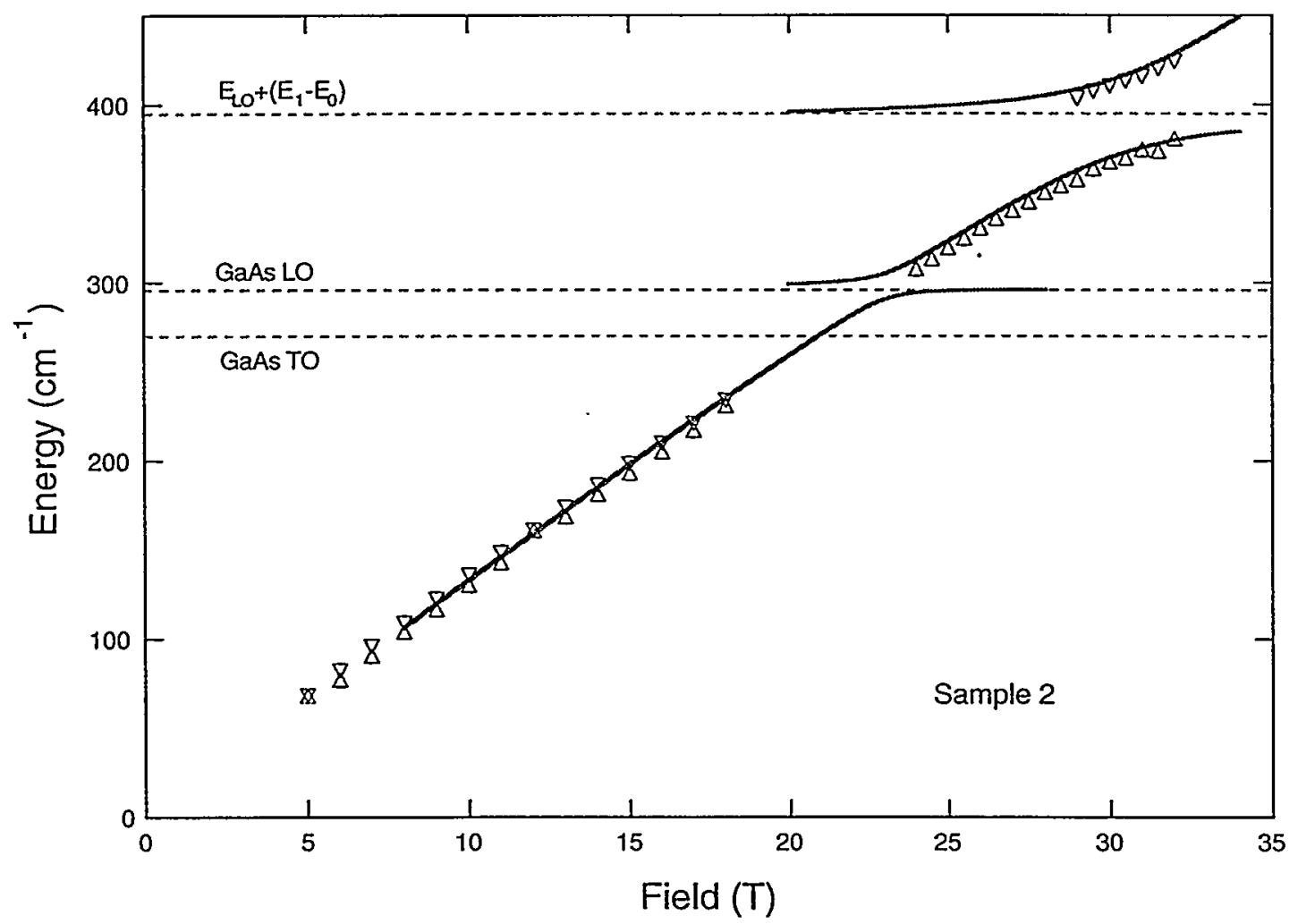

Figure 1. Measured electron $\mathrm{CR}$ frequency vs. magnetic field for the two samples. The solid lines are the calculated single particle electron $C R$ transition energies for the $N=0$ to $\mathrm{N}=1$ transition (ignoring spin) of the ground subband including non-parabolicity. 

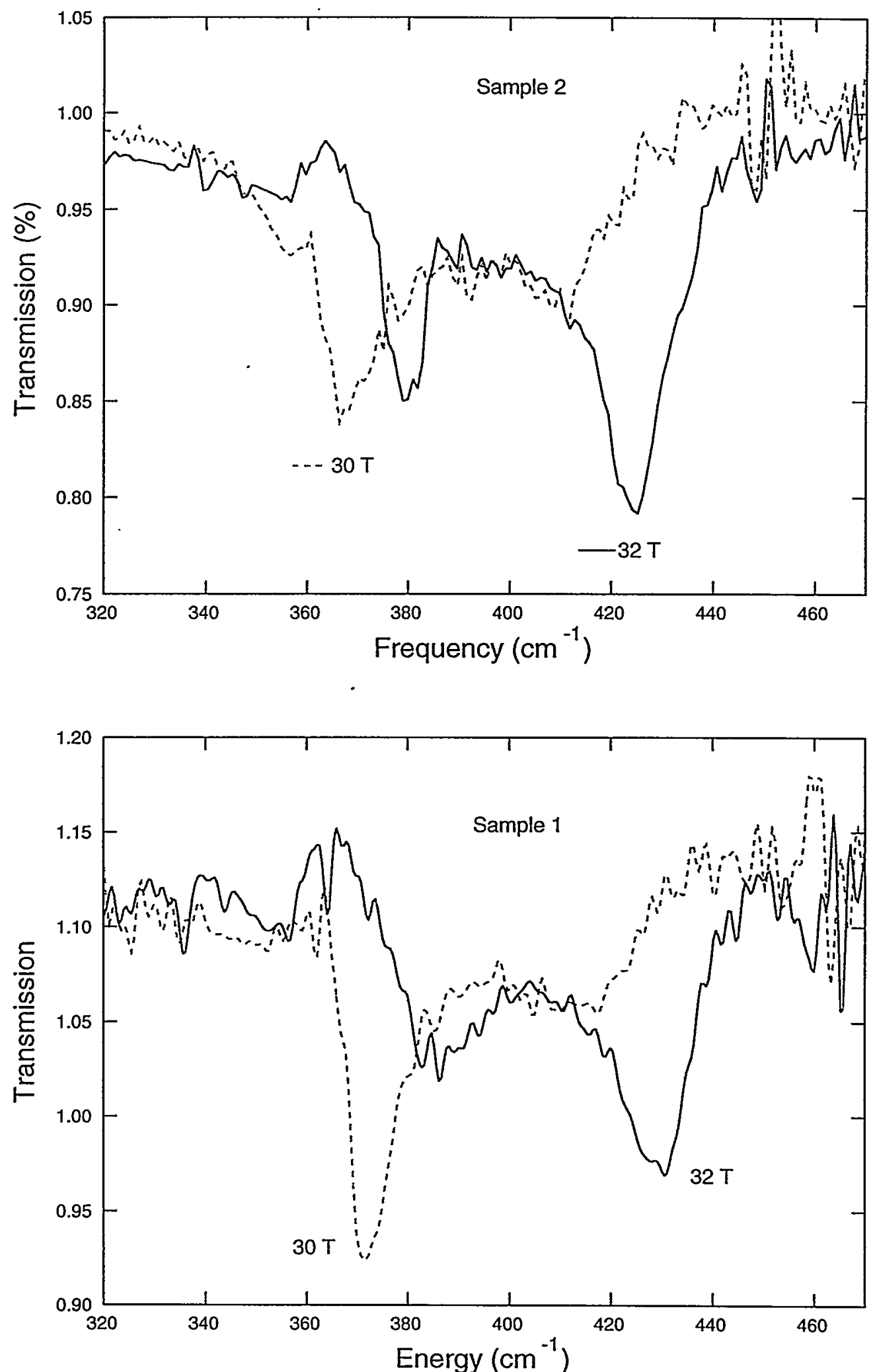

Figure 2. Magnetotransmission spectra for these two samples at $30 \mathrm{~T}$ and $32 \mathrm{~T}$. These spectra are normalized magnetotransmission, ratios of a spectrum taken at magnetic field to a spectrum taken at zero field. 